\title{
Optically Transparent Focused Transducers for Combined Photoacoustic and Ultrasound Microscopy
}

\author{
Sunghun Park ${ }^{1}$. Sungwoo Kang ${ }^{1} \cdot$ Jin Ho Chang $^{2}$ (i)
}

Received: 3 March 2020 / Accepted: 20 May 2020 / Published online: 26 May 2020

(c) The Author(s) 2020

\begin{abstract}
Purpose Photoacoustic (PA) microscopy has emerged as a useful tool in biomedical imaging applications such as visualization of microvasculature and hemoglobin oxygen saturation, single-cell, and label-free imaging of organs including cancer. Since the ultrasound transducers used for PA signal detection are not optically transparent, the integration of optical and acoustic modules for coaxial alignment of laser and acoustic beam fields in PA microscopy is complex and costly.

Methods Here, we report a recently developed optically transparent focused transducer for combined PA and ultrasound (US) microscopy. All the acoustic layers including the acoustic lens are optically transparent, enabling simple integration of optical and acoustic modules for both imaging modalities.

Results The mean light transmittance of the transducer's backing layer and acoustic lens and of the transducer itself were measured at $92 \%, 83 \%$, and $66 \%$, respectively. Results from in vitro and in vivo experiments demonstrated the transducer to be suitable for both US and PA imaging.

Conclusions The results of this study represent a step toward efficient construction of probes for combined PA and US microscopy.
\end{abstract}

Keywords Optically transparent sensor · Photoacoustic signal detection · Photoacoustic microscopy · Ultrasound imaging · Hybrid imaging

\section{Introduction}

Photoacoustic microscopy (PAM) has emerged as a useful tool in biomedical imaging applications due to its ability to provide anatomical and functional information with high spatial and contrast resolution. It can be used for imaging of microvasculature and hemoglobin oxygen saturation, single-cell imaging, and label-free imaging of organs including cancer [1-5]. Photoacoustic (PA) signals are generated from chromophores irradiated with laser and detected using an ultrasound (US) transducer. Hence, high-quality PAM imaging demands more sophisticated integration of optical and acoustic modules for coaxial alignment of the laser and

Jin Ho Chang

jhchang@dgist.ac.kr

1 Department of Electronic Engineering, Sogang University, Seoul 04107, Korea

2 Department of Information and Communication Engineering, Daegu Gyeongbuk Institute of Science and Technology, Daegu 42988, Korea acoustic beam fields than cross-section PA imaging [6, 7]. Initially, an integration scheme based on dark-field illumination was used; an approach suitable for acoustic-resolution PAM (AR-PAM) [8] but not for optical-resolution PAM (OR-PAM). Use of a ring-shaped transducer enables removal of the optically reflective mirror placed around the transducer in dark-field illumination, supporting a more compact design suitable for OR-PAM [3, 9]. Since irradiating laser light can propagate through the opening in the transducer, laser energy loss is minimized. However, this configuration limits the lateral resolution of PA images because the ringshaped aperture leads to an increase in sidelobe levels [10, $11]$, reducing image quality, particularly in terms of contrast resolution [12]. In addition, the central hole has been reported to cause acoustic energy loss of greater than $10 \%$ [13]. Therefore, ring-shaped transducers may not be the optimal solution for AR-PAM. Development of optical-acoustic beam combiners, using either acoustic reflectors $[14,15]$ or optical reflectors [16], has facilitated OR-PAM using scanning mirrors. However, this requires acoustically and optically sophisticated designs, and a considerable acoustic loss 
occurs when acoustic reflectors are employed. Note that all these configurations have arisen from the fact that US transducers are optically opaque.

An US imaging transducer consists of an active layer (i.e., piezoelectric material), acoustic matching layers, a backing layer, and an acoustic lens $[6,17]$. The active layer is generally plated with gold to enable transmission of electrical signals to and from the imaging system. If this gold electrode is replaced with indium tin oxide (ITO), an optically transparent active layer can be created; optically transparent active layers based on lithium niobate $\left(\mathrm{LiNbO}_{3}\right)$ have been demonstrated previously [18]. Note that ITO has a light transmittance greater than $80 \%$, although its exact value depends on deposition techniques [19]. In addition, optically transparent capacitive micromachined ultrasonic transducers (CMUTs) have been reported $[20,21]$. Recently, it has been demonstrated that a $\mathrm{LiNbO}_{3}$-based transparent transducer can be used for OR-PAM [22, 23]. However, there are still technical barriers to overcome in AR-PAM and combined US and PA imaging: The acoustic lens is a key component for both imaging modes, but typical acoustic lenses are constructed from optically opaque materials. For US imaging, geometric focus is a viable alternative to acoustic lenses [24]. However, this may be unsuitable for optically transparent transducers based on single crystal materials such as $\mathrm{LiNbO}_{3}$ since such materials are prone to developing cracks during geometric focus fabrication, which can hinder light penetration. Alternatively, polyvinylidene fluoride (PVDF), another transparent piezoelectric material, offers improved flexibility, and a PVDF-based transparent transducer has recently been developed for PAM [25]. This material is a good choice for PAM because it supports easy shaping for focus and a relatively broad bandwidth, but is not suitable for combined PA and US microscopy because of its low electromechanical coupling coefficient and high mechanical and electrical losses compared to other piezoelectric materials, resulting in low sensitivity [26]. This is the main reason why PVDF is not commonly used for US imaging. Note that both AR-PAM and OP-PAM can be used for imaging targets located in the focal area of an US transducer.

Combined PA and US imaging enables simultaneous acquisition of anatomical, functional, and molecular information from the imaging target area [27-29]. Combined PA and US microscopy offers the same benefits [30, 31]. Here, we report a recently developed optically transparent focused US transducer that can be used for combined PA and US microscopy. The transducer consists of an active layer, a backing layer, and an acoustic lens, which were constructed using optically transparent materials. In this paper, the design and fabrication process of the transducer for both PA and US imaging is described. The acoustic and optical characteristics of the transducer are presented, and its imaging performance evaluated through in vitro and in vivo experiments. The experimental results demonstrate that the proposed design and fabrication process for optically transparent US transducers can be used for efficient construction of probes for AR-PAM and combined PA and US microscopy.

\section{Design and Fabrication}

For an optically transparent focused US transducer, Y-36 ${ }^{\circ}$ cut $\mathrm{LiNbO}_{3}$ (Boston Piezo-Optics, Bellingham, MA, USA) was used as the active layer (Fig. 1a). This material is known to pass $70 \%$ of irradiated light with a wavelength in 300-1000 $\mathrm{nm}$ range [18]. The thickness and size of the $\mathrm{LiNbO}_{3}$ were designed as $280 \mu \mathrm{m}$ and $7 \mathrm{~mm} \times 7 \mathrm{~mm}$, respectively, using finite element analysis in PZFlex software (Weidlinger Associates, Los Altos, CA, USA). Note that the thickness of the designed transducer corresponds to a center frequency of $11 \mathrm{MHz}$ theoretically. Epoxy (Epo-tek 301, Epoxy technologies, Billerica, MA, USA) was used for the acoustic lens and backing layer due to its optical transparency: It has a light transmittance of $95 \%$ in the $382-1640 \mathrm{~nm}$ range. Since sound travels faster through epoxy than tissue, the radius of curvature of the acoustic lens was calculated using [32].

$R_{C}=F_{d}\left(1-\frac{V_{M}}{V_{L}}\right)$

where $V_{M}$ and $V_{L}$ are the speed of sound in tissue $(1500 \mathrm{~m} / \mathrm{s}$ ) and the epoxy $(2650 \mathrm{~m} / \mathrm{s})$, respectively. Since the active layer was determined to have a focal length of $11 \mathrm{~mm}$ (i.e., $F_{d}=$ $11 \mathrm{~mm}$ ), the radius of curvature was calculated as $4.77 \mathrm{~mm}$, but for ease of fabrication, this was set as $5 \mathrm{~mm}$, changing the focal length of the $\mathrm{LiNbO}_{3}$ to $11.52 \mathrm{~mm}$. The F-number of the active layer, defined as the ratio of focal length to the diagonal length of the aperture, was 1.16. We designed the lens edge to be $5 \mathrm{~mm}$ thicker than the center, to serve as the standoff for the transducer. As a result, the transducer's focal point was $6.52 \mathrm{~mm}$, accounting for the standoff. The backing layer was designed for easy integration with an optical fiber bundle; it had a flat surface with a thickness of $7 \mathrm{~mm}$, and the inner diameter of the housing was $10 \mathrm{~mm}$, similar to the outer diameter of the optical fiber bundle used in this study.

The fabrication process began by fixing the bulk $\mathrm{LiNbO}_{3}$ measuring $20 \mathrm{~mm} \times 20 \mathrm{~mm} \times 1 \mathrm{~mm}$ onto a glass plate using paraffin wax. The bulk $\mathrm{LiNbO}_{3}$ was lapped down to a target thickness of $280 \mu \mathrm{m}$, and then sputtered with an ITO of 1100 $\AA$ as electrodes, and diced to a size of $7 \mathrm{~mm} \times 7 \mathrm{~mm}$. For fabrication of the acoustic lens, a hemispherical steel ball with a $5 \mathrm{~mm}$ radius was fixed onto a glass plate, and a brass cylinder with an inner diameter of $14 \mathrm{~mm}$ was placed on the steel ball. Epo-tek 301 was poured into the brass cylinder 
Fig. 1 a Schematic cross section and $\mathbf{b}$ photograph of the optically transparent focused ultrasound transducer developed for both PA and US imaging; c Photograph showing light propagating through the transducer
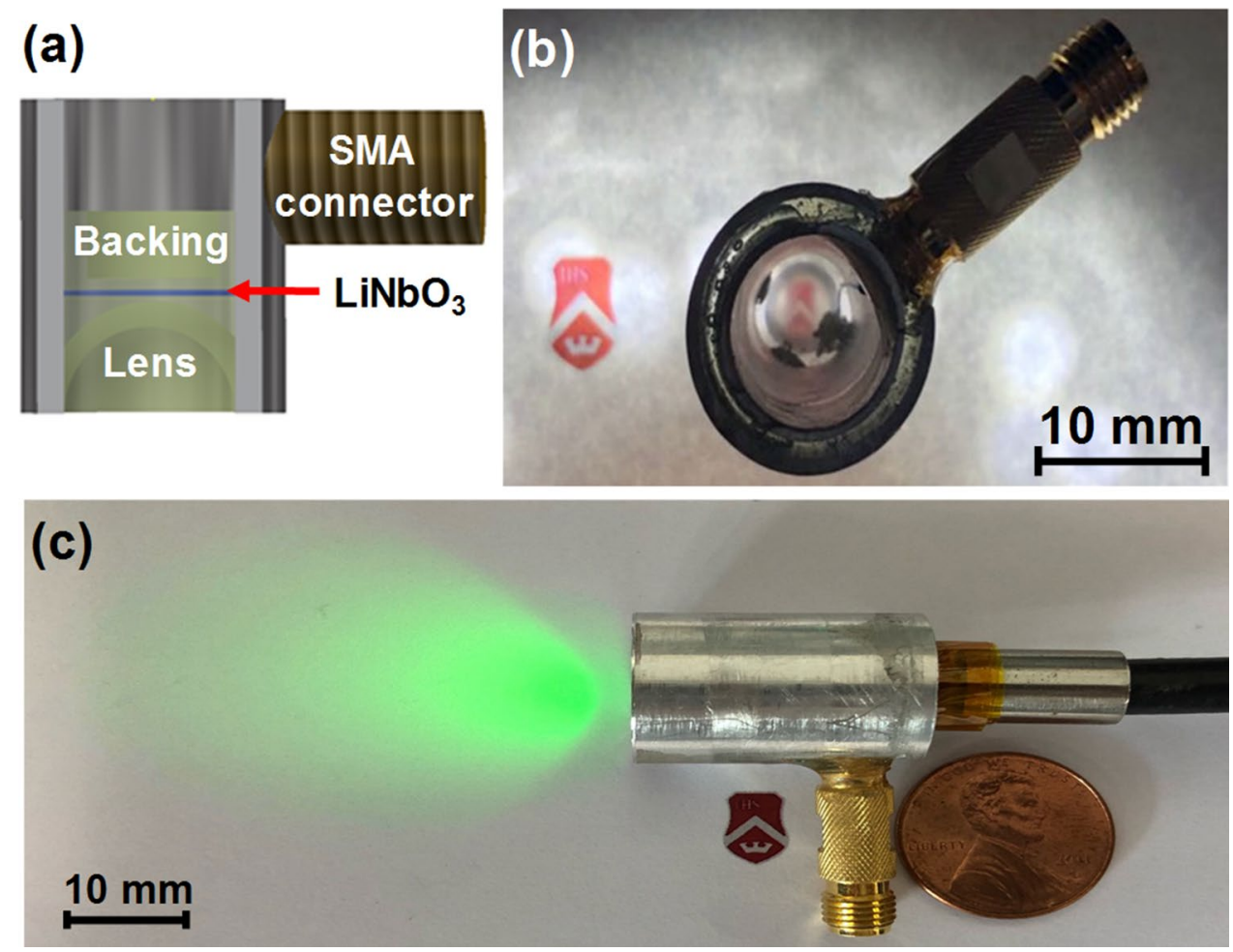

and cured for $24 \mathrm{~h}$ at a room temperature. The acoustic lens was bonded onto the prepared $\mathrm{LiNbO}_{3}$. Then, the backing layer was constructed using Epo-tek 301, having a flat surface with a thickness of $7 \mathrm{~mm}$. The backing layer was also bonded onto the $\mathrm{LiNbO}_{3}$. Figure $1 \mathrm{~b}$ shows photographs of the finished US transducer. Fabricating the transducer using optically transparent acoustic layers enabled light to propagate through the resulting device (Fig. 1c).

\section{Methods}

\subsection{Acoustic Characterization}

A pulse-echo response was obtained to evaluate the center frequency and spectral bandwidth of the transducer. For this, a stainless steel reflector was placed in a container filled with deionized and degassed water. The transducer was immersed in the container, and the focal point of the transducer was located on the reflector by adjusting the distance between the transducer and the reflector. The distance at which the maximum pulse-echo response was obtained was accepted as the focal point of the transducer. In this experiment, a pulse receiver system (UT340, UTEX Scientific Instruments Inc., Mississauga, ON Canada) was used to excite the transducer and receive the echo from the reflector. The echo was digitized and recorded using a digital oscilloscope (DPO7054, Tektronix Inc., Beaverton, OR, USA). The center frequency and spectral bandwidth of the echo were computed on MATLAB (Mathworks Inc., Natick, MA, USA). Additionally, the electrical impedance of the transducer was measured using an impedance analyzer (HP4294A, Agilent Technologies Inc., Santa Clara, CA, USA).

The spatial resolution of the transducer was also assessed. For this, the beam profile of the transducer was estimated by finite element analysis using the PZFlex. In addition to this simulation, the beam profile was measured using an acoustic intensity measurement system (AIMS) (ONDA Corp., Sunnyvale, CA, USA) equipped with a needle hydrophone (HGL-0085). For the measurement, 5-cycle sinusoidal waves with a frequency of $11.2 \mathrm{MHz}$ and an amplitude of $10 \mathrm{~V}_{\mathrm{p}-\mathrm{p}}$ were generated using a function generator (AFG3102C, Tektronix Inc., Beaverton, OR, USA) and sent to the transducer to transmit US. Additionally, insertion loss was measured to assess the transducer's sensitivity. For this, the stainless steel reflector was placed at the focal length of the transducer immersed in the water-filled container. A 20-cycle sinusoidal wave burst with a frequency of $11.2 \mathrm{MHz}$ and an amplitude of 5 $\mathrm{V}_{\mathrm{p}-\mathrm{p}}$ generated using the function generator was applied to the transducer. The voltage corresponding to the echo signals received by the transducer was recorded using the oscilloscope in $1 \mathrm{MHz}$ coupling mode. The ratio of the voltage generated by the transducer to the voltage applied to the transducer was calculated on a dB scale. A detailed experimental setup for the insertion loss measurement can be found in [26]. 


\subsection{Light Attenuation Measurement}

The attenuation of light energy in each acoustic layer was measured using a laser power meter (Maestro, Gentec-EO Inc., Quebec, Canada). For this, an optical fiber bundle was mounted on a motorized 3-axis XYZ stage (SGSP26-100, SIGMAKOKI Co. Ltd., Tokyo, Japan). As a reference, the laser power meter was placed at the surface of the optical fiber bundle and light emitted with an energy of $10.5 \mathrm{~mJ} /$ $\mathrm{cm}^{2}$ was measured in air. During the measurement, the laser wavelength was changed from 690 to $910 \mathrm{~nm}$ at $20 \mathrm{~nm}$ intervals. An Nd:YAG laser excitation system (Surelite III-10 and Surelite OPO Plus, Continuum Inc., Santa Clara, CA, USA) was used to deliver laser through the optical fiber bundle. The light energy was measured after placing the acoustic module directly in front of the optical fiber bundle. Note that the laser power meter was also placed on the surface of each acoustic module. The measured energy was normalized by the reference value to eliminate the wavelength-dependent fluctuation of light energy generated from the Nd:YAG laser system. In addition, the distribution of the 730-nm laser was measured to observe beam spreading caused by the acoustic lens after sequentially placing a laser screen at a distance of $6 \mathrm{~mm}$ from the optical fiber bundle (i.e., with no acoustic module), the backing layer, the acoustic lens, and the transducer. Note that the transducer's focal point was $6 \mathrm{~mm}$. The laser beam profile on the screen was measured using a charge-coupled device (CCD) camera (CoolSNAP MYO, Photometrics, Tucson, AZ, USA).

\subsection{Imaging Performance Evaluation}

For PAM imaging, the transducer was mounted on the motorized 3-axis XYZ stage, and the Nd:YAG laser excitation system was used to deliver laser through the optical fiber bundle, as shown in Fig. 2. The laser had a length of $7 \mathrm{~ns}$, a wavelength of $730 \mathrm{~nm}$, a pulse repetition rate of $10 \mathrm{~Hz}$, and an energy density of $14 \mathrm{~mJ} / \mathrm{cm}^{2}$. Whenever laser pulses were produced, the Nd:YAG laser source generated a trigger signal. The pulse receiver system started receiving PA signals in synchronization with the trigger signal. The PA signal was amplified by $35 \mathrm{~dB}$ in the pulse receiver system and sent to a Gage card (CS1422, Gage Applied Technologies Inc., Montreal, QC, Canada). The PA signal received by the Gage card was digitized at a sampling frequency 200 $\mathrm{MHz}$ and recorded. The signal acquisition was controlled by a program written in LabVIEW (National Instruments, Austin, TX, USA). After recording one scanline of data, the transducer was moved by a given distance using the motorized stage controlled by the LabVIEW program: $10 \mu \mathrm{m}$ for gold-wire imaging, $40 \mu \mathrm{m}$ for silicone tube imaging, and $20 \mu \mathrm{m}$ for mouse tail imaging. For US imaging, the pulse receiver system was used to excite the transducer and receive echoes.

A gold wire with a diameter of $25 \mu \mathrm{m}$ was used to evaluate the transducer's spatial resolution, as shown in Fig. $2 b$. The wire was immersed in a container filled with deionized and degassed water. The transducer's position was adjusted using the motorized stage to place the wire at the focal point of the transducer. To evaluate imaging performance, we prepared silicone tubes (AAQ04091, Tygon ${ }^{\circledR}$ Medical Tubing, Saint-Gobain Corp.) with inner and outer diameters of $250 \mu \mathrm{m}$ and $760 \mu \mathrm{m}$, respectively. Blue ink was injected into the tubes immersed in the container. For PA imaging, laser pulses with a length of $7 \mathrm{~ns}$ and an energy density of $12 \mathrm{~mJ} / \mathrm{cm}^{2}$ were used. The laser wavelength was $720 \mathrm{~nm}$, at which the laser energy was maximally absorbed by the blue ink. US and PA images with a size of $12 \mathrm{~mm} \times 12 \mathrm{~mm}$ (a)

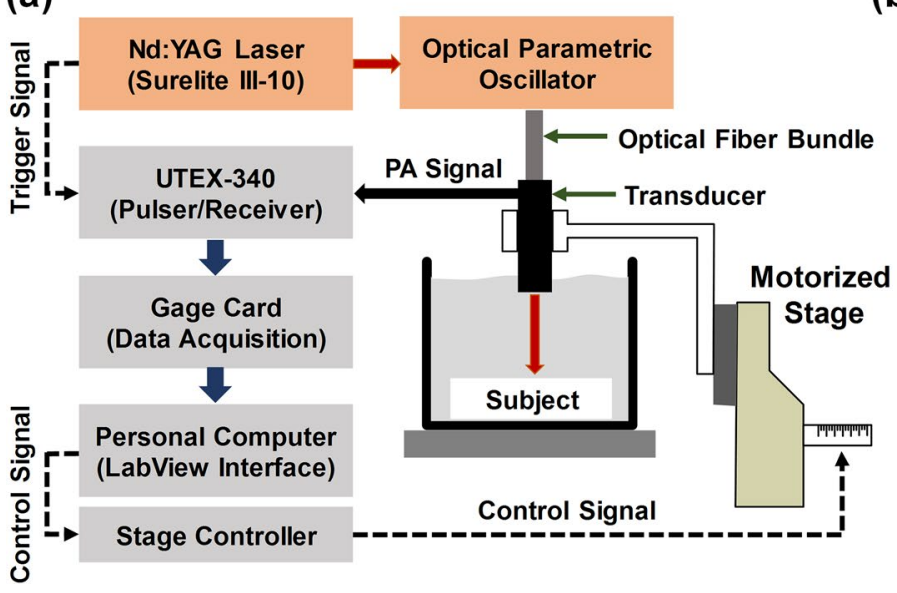

(b)

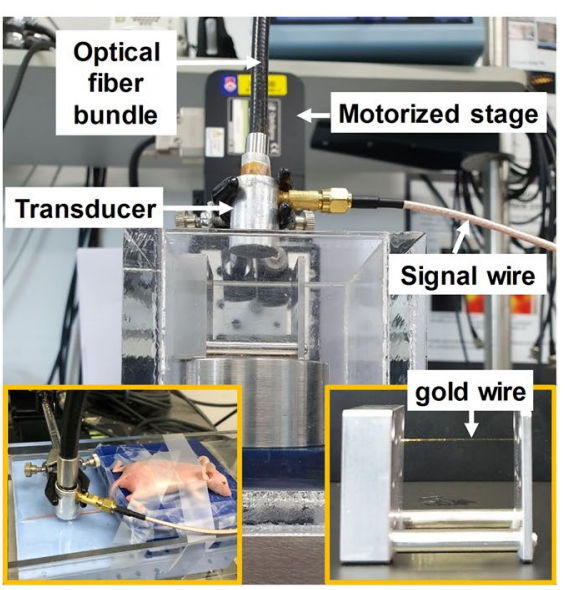

Fig. 2 Experimental setup. a Block diagram and $\mathbf{b}$ photograph of the experimental setup for the in vitro and in vivo ultrasound and photoacoustic imaging using the developed transducer 
were acquired, which were logarithmically compressed with a dynamic range of $20 \mathrm{~dB}$ and $10 \mathrm{~dB}$ for the US and PA images, respectively.

The in vivo US and PA imaging of a 5-week-old female $\mathrm{BALB} / \mathrm{c}$ mouse tail was performed. This experiment was conducted in accordance with the guidelines and regulations approved by the Institutional Animal Care and Use Committee at Sogang University. The mouse was placed on an acoustic absorption pad (Fig. 2b) and anesthetized by intraperitoneal injection of avertin $(0.4 \mathrm{~mL})$. For in vivo imaging, laser pulses with a length of $7 \mathrm{~ns}$, an energy density of 14 $\mathrm{mJ} / \mathrm{cm}^{2}$, and a wavelength of $680 \mathrm{~nm}$ were used. US and PA images of size $5 \mathrm{~mm} \times 10 \mathrm{~mm}$ were acquired, and then logarithmically compressed with a dynamic range of $40 \mathrm{~dB}$ and $10 \mathrm{~dB}$ for the US and PA images, respectively.

\section{Results}

In the pulse-echo test, the developed transducer was found to have a center frequency of $11.2 \mathrm{MHz}$ and a $-6 \mathrm{~dB}$ fractional bandwidth of $23 \%$ (i.e., 9.9 to $12.5 \mathrm{MHz}$ ), as shown in Fig. 3a. Note that the pulse-echo appeared at a time corresponding to the focal length of the $\mathrm{LiNbO}_{3}$, i.e., about 11 $\mathrm{mm}$. The magnitude and phase angle of the electrical impedance of the developed transducer were $245 \Omega$ and $-25.6^{\circ}$, respectively, at the center frequency (see Fig. 3b). The transducer's focal point was measured at $6 \mathrm{~mm}$, corresponding to the distance between the transducer's standoff and the reflector when the maximum echo amplitude was obtained. The beam profile measurement using the AIMS confirmed the focal point to be $6 \mathrm{~mm}$, as shown in Fig. 3d, e. Also, the $-3 \mathrm{~dB}$ lateral beam width and depth of focus (DOF) were $185 \mu \mathrm{m}$ and $1.4 \mathrm{~mm}$ (i.e., from 5.5 to $6.9 \mathrm{~mm}$ ), respectively, in good agreement with the simulation results (i.e., a focal point of $5.96 \mathrm{~mm}, \mathrm{a}-3 \mathrm{~dB}$ lateral beam width of $182 \mu \mathrm{m}$, and a DOF of $1.78 \mathrm{~mm}$; see Fig. 3c). The transducer's insertion loss was $17.12 \mathrm{~dB}$ at a center frequency of $11.2 \mathrm{MHz}$.

Although the acoustic layers are optically transparent, some attenuation of light energy was expected to occur in each layer. This was therefore measured as a function of wavelength in the 690-910 nm range (see Fig. 4a). As a reference, light energy was measured in air after placing the laser power meter at the surface of the optical fiber bundle emitting laser light with an energy density of 10.5 $\mathrm{mJ} / \mathrm{cm}^{2}$; the light distribution had a Gaussian beam profile with a full-width at half-maximum (FWHM) of $6.86 \mathrm{~mm}$. The CCD camera placed at the focal depth of the transducer (i.e., $6 \mathrm{~mm}$ ) was used to measure the light distribution (see top left panel of Fig. 4b, c). The light energy after propagating through each acoustic layer was measured and normalized by the reference value because light energy
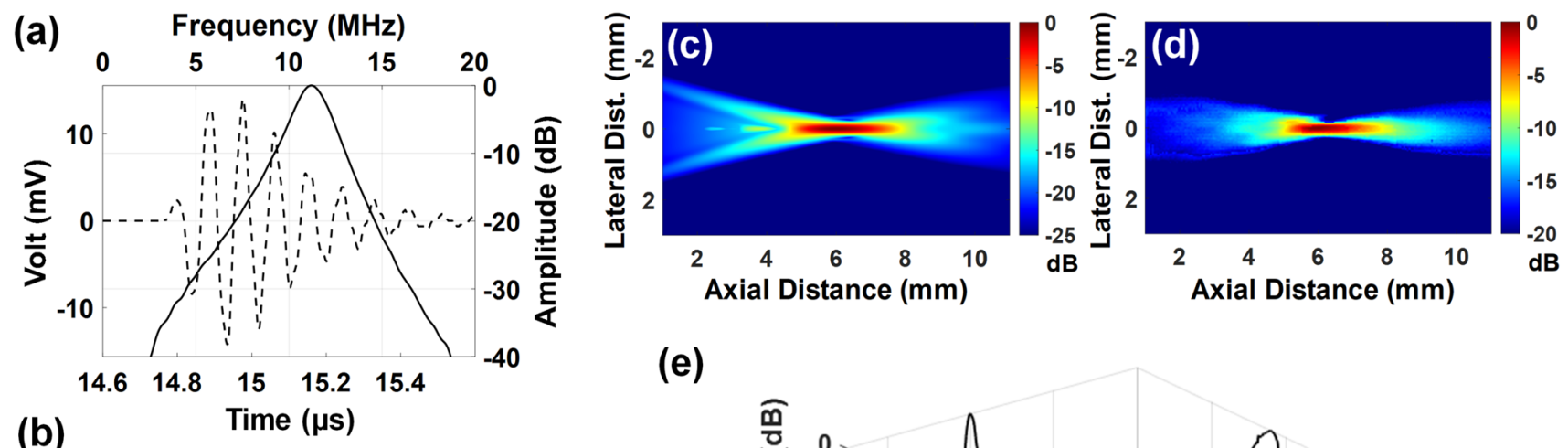

(e)
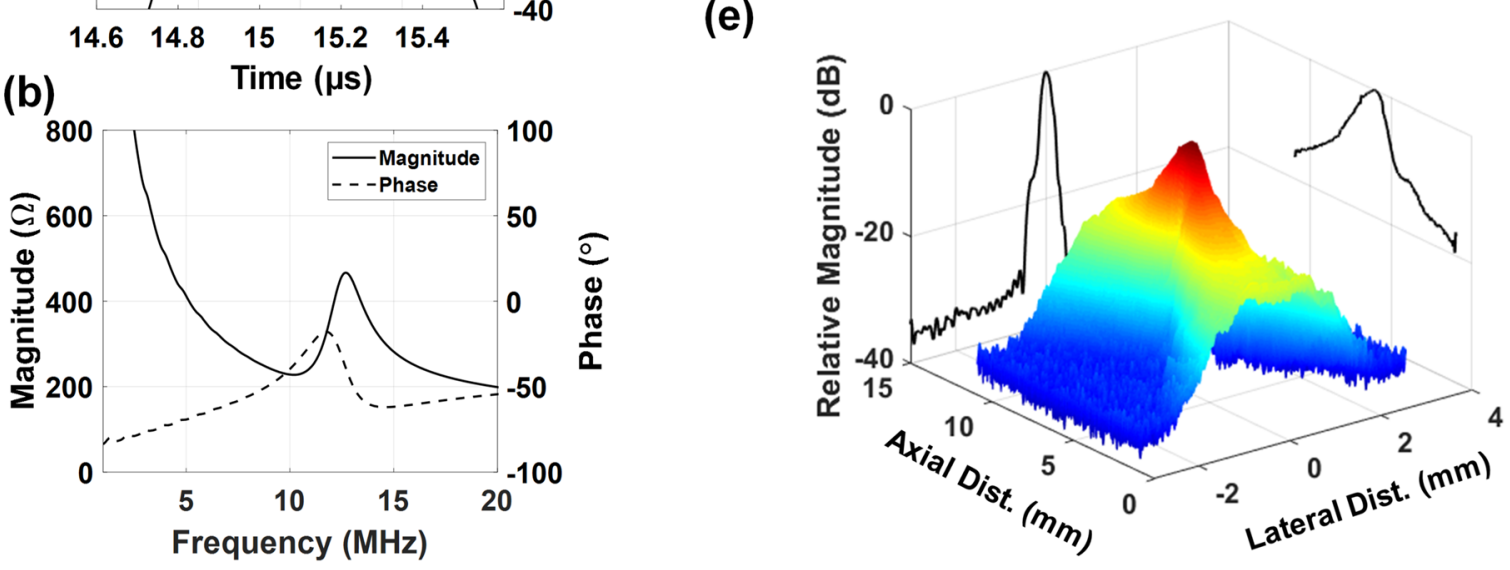

Fig. 3 a Measured pulse-echo response (dashed line) and its frequency spectrum (solid line) and $\mathbf{b}$ measured electrical impedance magnitude (solid line) and phase angles (dashed line). Ultrasound

beam profile measurement: $\mathbf{c}$ simulated and $\mathbf{d}$ measured beam profiles of the developed transducer; e measured lateral and axial ultrasound beam profiles at a focal point of $6 \mathrm{~mm}$ 

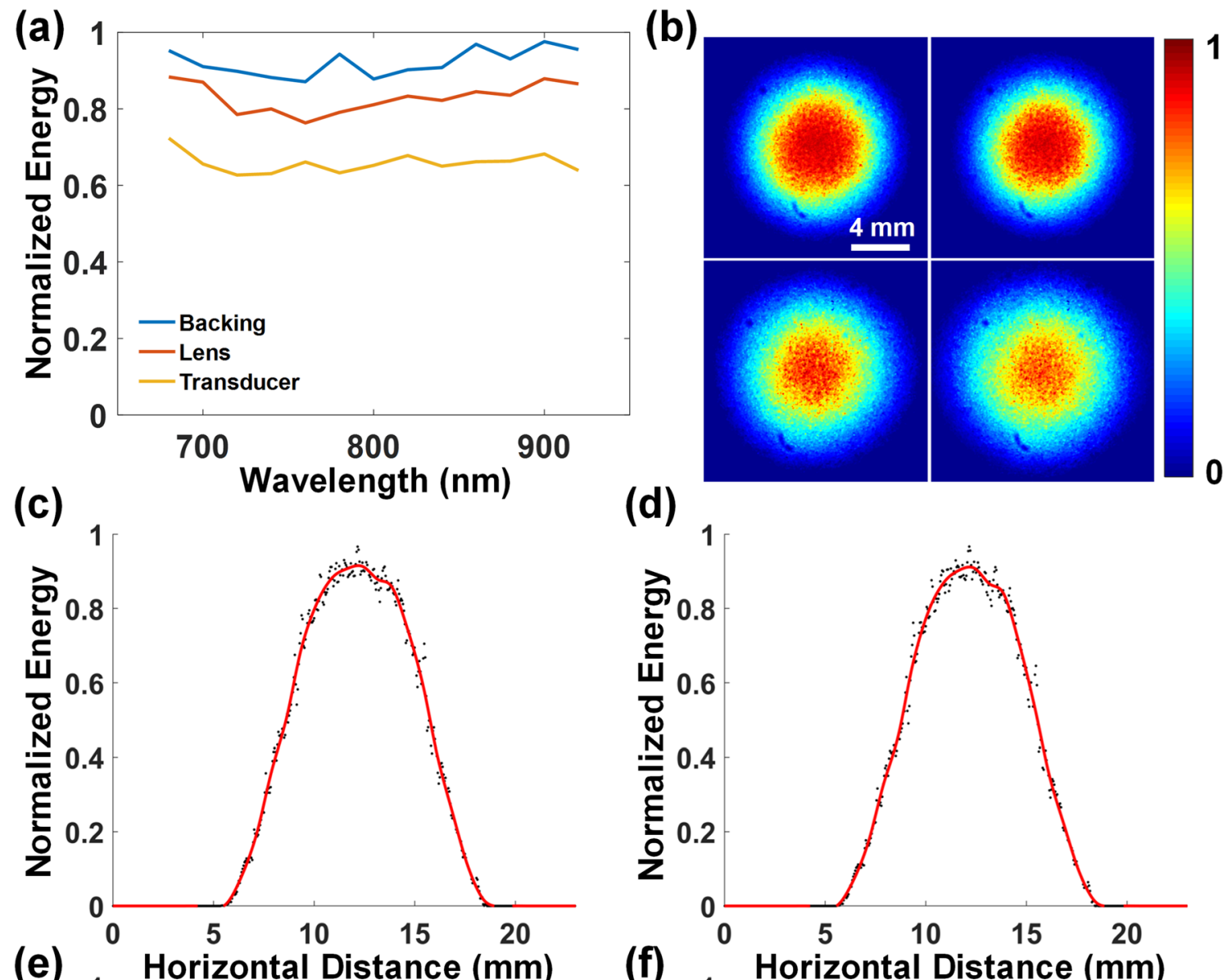

(d)
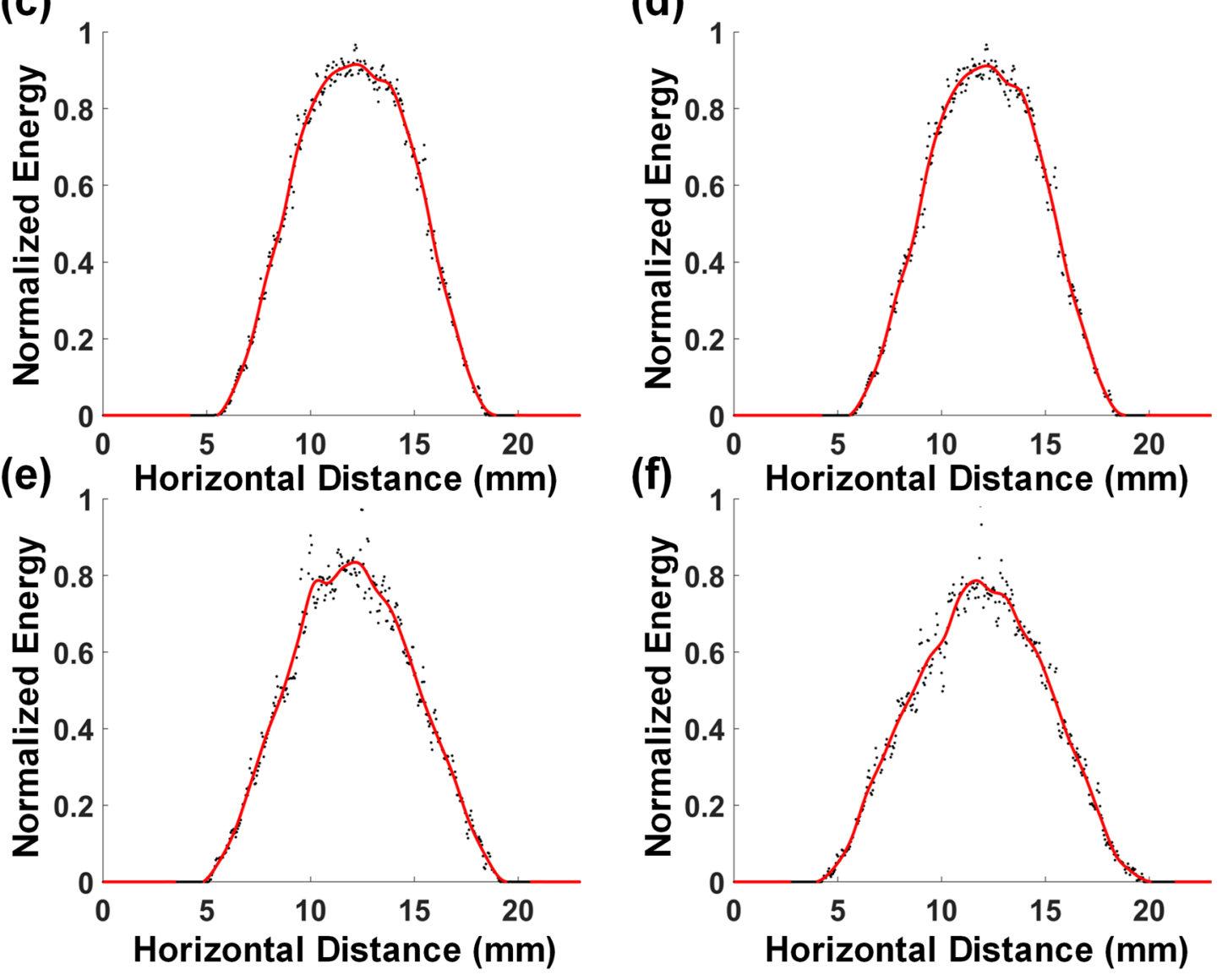

Fig. 4 a Mean light attenuation occurring after penetrating each acoustic layer; $\mathbf{b}$ light distributions measured at the focal point of the transducer (i.e., $6 \mathrm{~mm}$ ) after penetrating no acoustic layers (top left), the backing layer (top right), the acoustic lens (bottom left), and the

from the Nd:YAG laser system used in this study varied with wavelength. The backing layer caused a mean energy loss of $8 \%$. Note that Epo-tek 301 has a light transmittance of $95 \%$ in the wavelength range of 382-1640 $\mathrm{nm}$. The FWHM of the light after passing through the backing layer was $6.70 \mathrm{~mm}$, similar to that of the reference.

transducer (bottom right); the distributions along the horizontal axis (dots) and the corresponding smoothing spline fitting curve (red line), measured after the irradiated light penetrated $\mathbf{c}$ no acoustic layers, $\mathbf{d}$ the backing layer, $\mathbf{e}$ the acoustic lens, and $\mathbf{f}$ the transducer

However, the light propagating through the acoustic lens spread considerably: FWHM was $7.14 \mathrm{~mm}$ and the normalized average light energy was 0.83 (i.e., an energy loss of $17 \%$ ) although the lens was made from Epo-tek 301. This was due to the plano-concave shape of the acoustic lens. As a result, the average light transmittance of the 
transducer was 66\%, and the FWHM of the light after the transducer was $7.21 \mathrm{~mm}$.

PA and US images of the $25-\mu \mathrm{m}$ gold wire were acquired to evaluate the spatial resolution of the transducer as shown in Fig. 5a and b, respectively. Axial beam profiles were obtained by vertically plotting the envelope of those images (see Fig. 5c); the $-3 \mathrm{~dB}$ and $-6 \mathrm{~dB}$ axial beam widths of the PA image were found to be 158 and $227 \mu \mathrm{m}$, respectively, whereas those of the US image were 148 and 224 $\mu \mathrm{m}$. Since the center frequency and fractional bandwidth of the transducer mainly determine axial resolution [33], it was reasonable for the PA and US images to have similar axial beam widths. On the other hand, the lateral beam width of the PA image was wider than that of the US image (Fig. 5d); the $-3 \mathrm{~dB}$ and $-6 \mathrm{~dB}$ lateral beam widths were 242 and 312 $\mu \mathrm{m}$ for the PA image, compared to 141 and $198 \mu \mathrm{m}$ for the US image.

To evaluate the imaging performance of the transducer, we prepared silicone tubes with inner and outer diameters of $250 \mu \mathrm{m}$ and $760 \mu \mathrm{m}$, respectively, as shown in Fig. 6a. The blue ink injected into the tubes maximally absorbed the laser with a wavelength of $720 \mathrm{~nm}$. In the US image (see top panel in Fig. 6b), the distance between the transducer and the tubes was measured at about 5.0, 6.5, 6.7, and $7.0 \mathrm{~mm}$; the PA image of the tubes at each depth was represented by orange, green, blue, and red (Fig. 6d). Since the DOF of the developed transducer ranged from 5.5 to $6.9 \mathrm{~mm}$, the tube located at a depth of $5.0 \mathrm{~mm}$ appeared larger than other tubes in both US and PA images. Both US and PA imaging methods were able to discriminate between the top and bottom of the tubes (Fig. $6 \mathrm{~b}$ ) because their axial beam widths were narrower than the tube size. As shown in Fig. 6e, the axial profiles of both US and PA images had two peaks. The distance between the two peaks in the PA image was measured at $247 \mu \mathrm{m}$, similar to the inner diameter of the silicone tube, i.e., $250 \mu \mathrm{m}$. The corresponding distance was $651 \mu \mathrm{m}$ for the US image. The large distance between the peaks in the US image was due to reflection of the transmitted US at the inner and outer boundaries of the tube. In contrast to the spatial resolution shown in Fig. 5, the US image had a wider lateral width than the PA image due to the US transmission; the -3 and $-6 \mathrm{~dB}$ lateral widths of the PA image
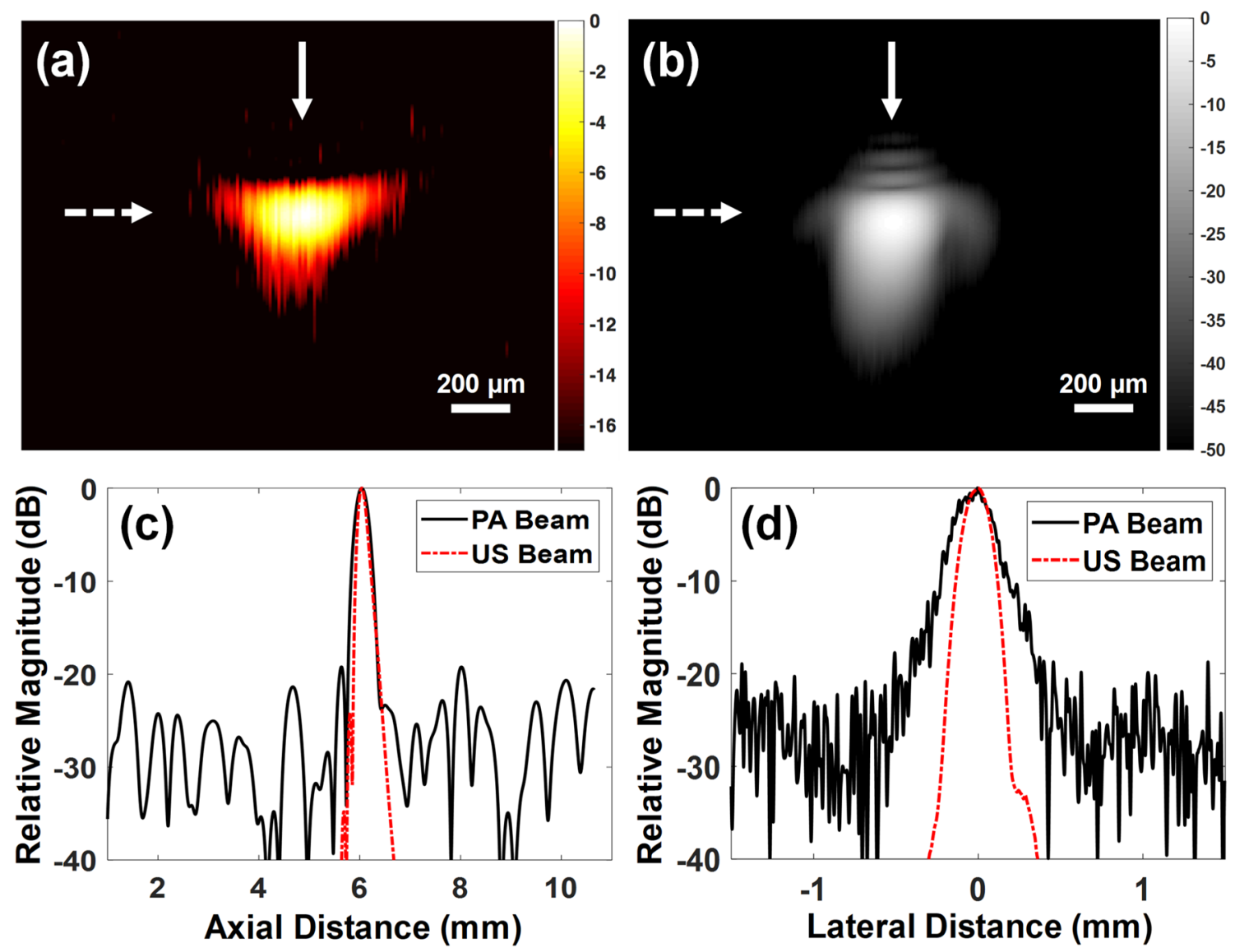

Fig. 5 Measurement of spatial resolution. a PA and b US images of a $25-\mu \mathrm{m}$ gold wire; c axial and $\mathbf{d}$ lateral beam profiles measured for quantitative analysis of the spatial resolution. The measurement

directions for the axial and lateral beam profiles are indicated by the solid arrows and the dashed arrows in (a) and (b), respectively. The color bars in (a) and (b) are in $\mathrm{dB}$ 
(a)

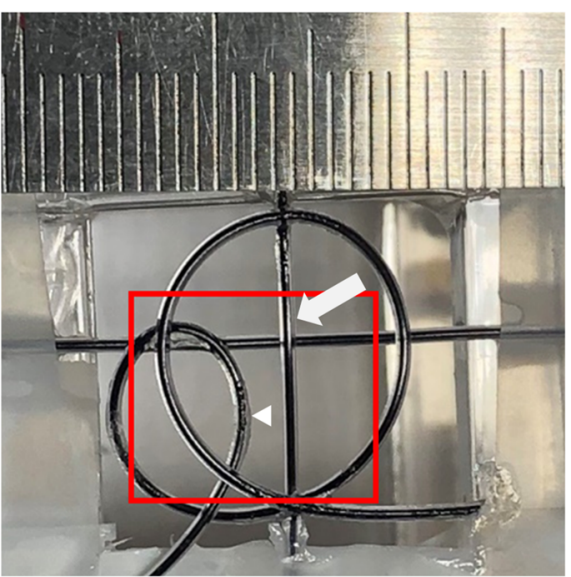

(c)

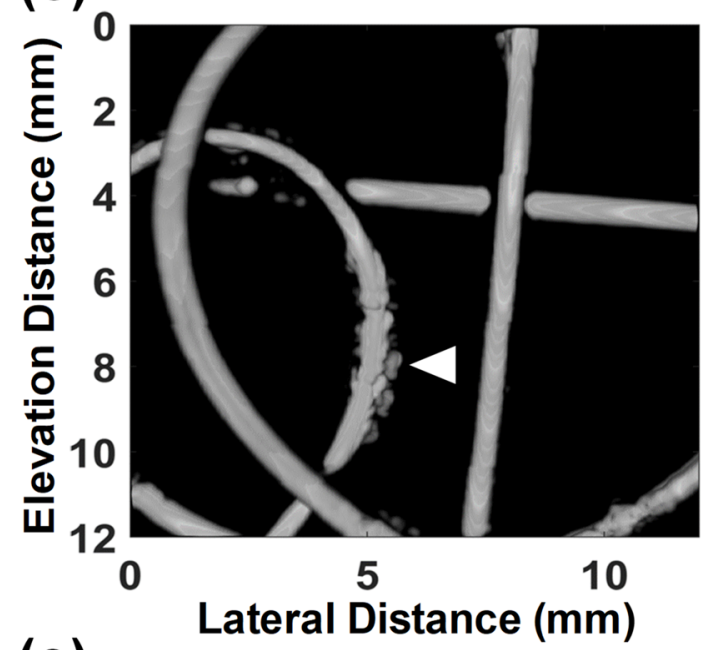

(e)

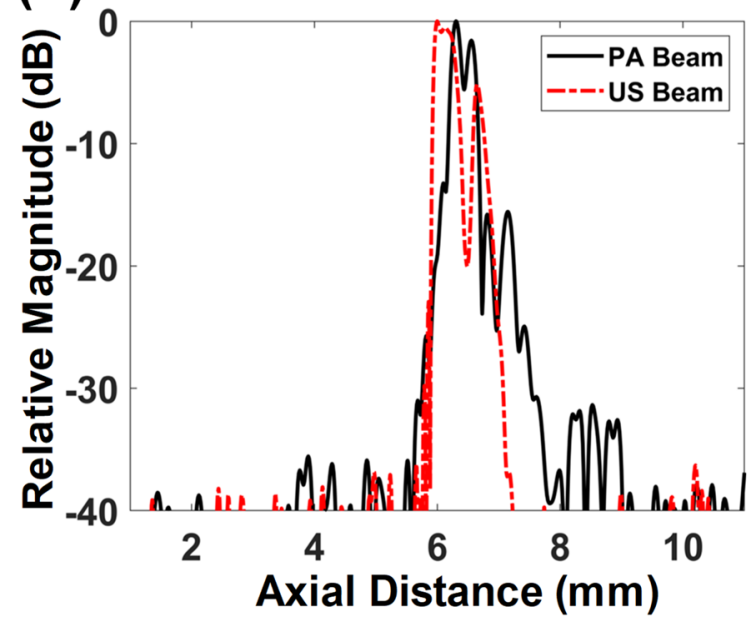

Fig. 6 Image performance evaluation. a Configuration of the blue ink-filled silicone tubes; b US (top) and PA (bottom) images in the axial-lateral imaging plane; $\mathbf{c}$ US and $\mathbf{d}$ PA maximum intensity projection images in the elevation-lateral imaging plane. e Axial and $\mathbf{f}$ lateral plots of the tube image indicated by the white arrow in (a). (b)
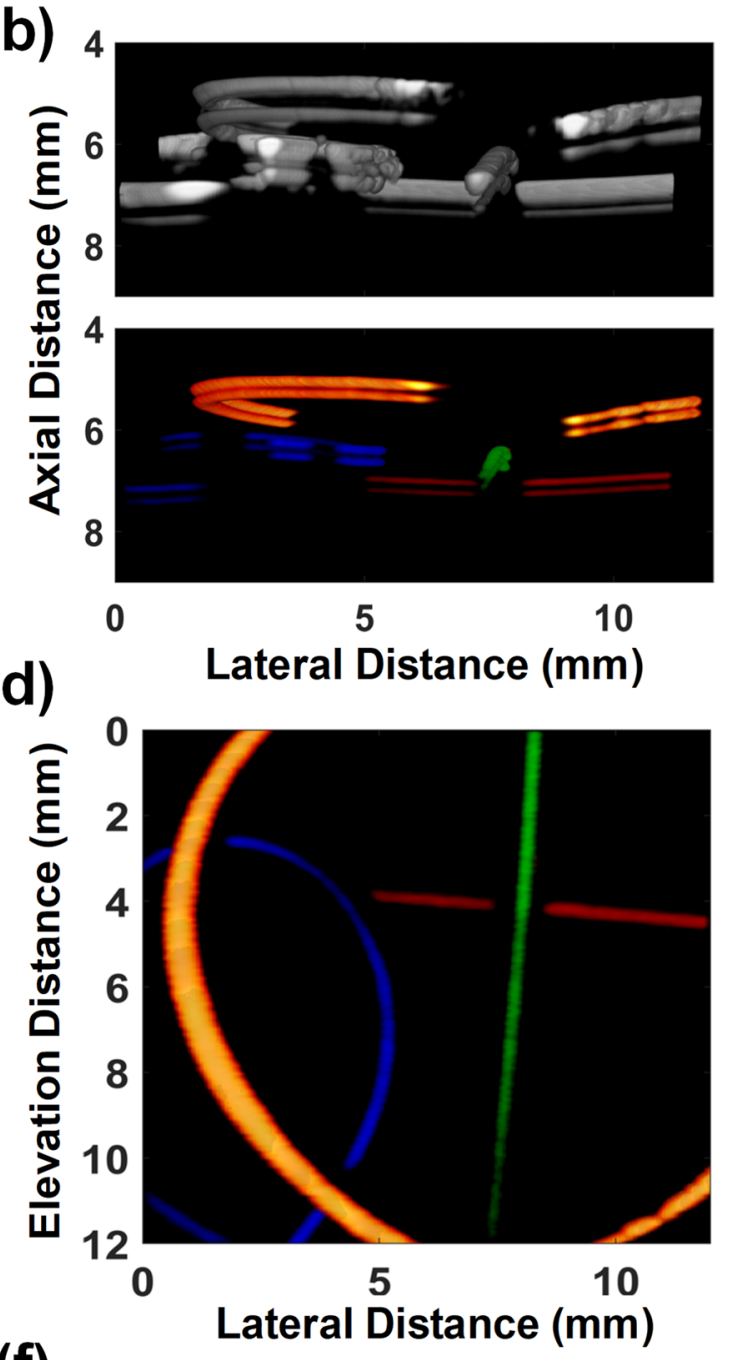

(f)

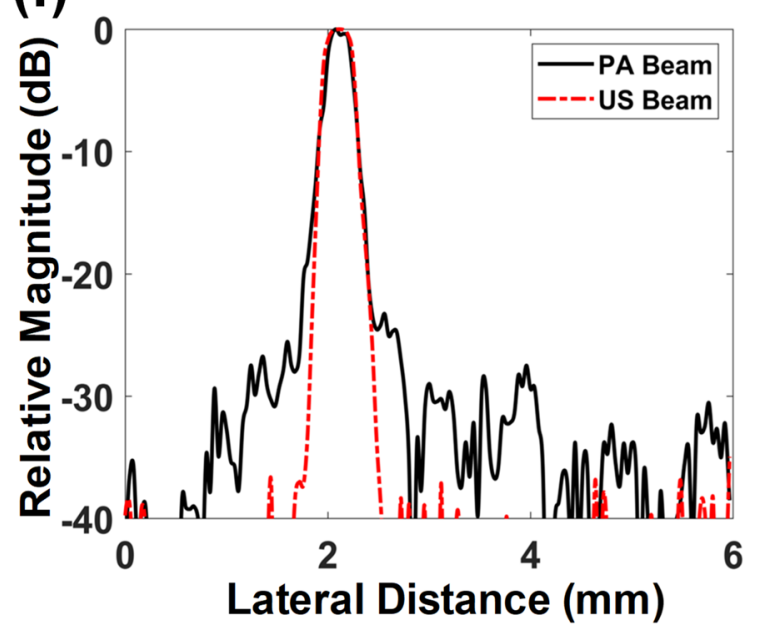

The US and PA images were logarithmically compressed with dynamic ranges of $20 \mathrm{~dB}$ and $10 \mathrm{~dB}$, respectively. The color in the PA images indicates the distance between the transducer and the tubes: $5.0 \mathrm{~mm}$ by orange, $6.5 \mathrm{~mm}$ by green, $6.7 \mathrm{~mm}$ by blue, and $7.0 \mathrm{~mm}$ by red 
were 244 and $308 \mu \mathrm{m}$, respectively, whereas those of the US image were 298 and $344 \mu \mathrm{m}$, respectively (Fig. 6f). Note that the lateral width of the US image differed considerably from the actual outer diameter of the tube because the incident US was reflected from the tube to the transducer only when the angle of incidence was small. This implies that the edge of the tube could not appear in the US image. The tube imaging also demonstrated the benefits of combined US and PA imaging: Structural and functional information about the target lesion can be obtained simultaneously from both imaging modalities [31, 34]. The US image represented the rough surface of the tube, indicated by the arrow head in Fig. 6a, c, whereas the PA image provided information about the spatial distribution of the blue ink.

The advantage of this fusion imaging can be also found in the US and PA images of the 5-week-old female BALB/c mouse tail. The US image delineated the shape of the mouse tail (see Fig. 7a, b), whereas the PA image provided information about tail veins (see Fig. 7c, d). The diameters of dorsal and lateral veins in a rat tail have been reported as $180 \mu \mathrm{m}$ and $400 \mu \mathrm{m}$, respectively [35], and the tail veins of a mouse are expected to be smaller than those of the rat. Hence, the tail veins did not appear in the US image because the beam profile of the transmitted US was not narrow enough to depict the vein structures. Conversely, the veins were captured by PA imaging because only the hemoglobin in the vessels could generate the PA signals. The combined US and PA images simultaneously provided structural and functional information about the mouse tail (see Fig. 7e, f). Note that Fig. $7 \mathrm{f}$ shows the combined PA and US image of the mouse tail in the elevation-lateral imaging plane (i.e., C-plane) at an axial depth of $6.43 \mathrm{~mm}$, indicated by the white arrow in Fig. 7e.

\section{Discussion}

A well-matched electrical impedance between a transducer and an imaging system is important for high-quality US and PA imaging. However, the electrical impedance of our transducer (i.e., $245 \Omega$ and $-25.6^{\circ}$ at the center frequency) was higher than commonly found in measurement instruments (i.e., $50 \Omega$ ), including those used for this study. An electrical impedance-matching network can be used to improve the efficiency of signal reception and/or transmission [36, 37]. When developing an imaging system for commercial purposes, however, the magnitude of the transducer's electrical impedance is not a problem because the input impedance of low noise amplifiers used for signal reception ranges from 50 to $300 \Omega$ [38]. Therefore, we did not perform electrical impedance matching between our transducer and the measurement instruments in this study. Despite this electrical impedance mismatch, the insertion loss of the developed transducer (i.e., $17.12 \mathrm{~dB}$ ) was comparable with that of $\mathrm{LiNbO}_{3}$-based single element transducers with similar aperture size, gold electrodes, and acoustic matching layers [17]. Note that the insertion losses of PVDF-based single element transducers are higher than $35 \mathrm{~dB}[26,39]$. This means that our transducer has sufficient sensitivity for combined PA and US imaging.

Although the developed transducer consisted of optically transparent materials with a light transmittance of higher than $80 \%$, the transducer had an average light transmittance of $66 \%$. One reason for this decrease in light transmittance is that the irradiated beam size was similar to the transducer's aperture size; the connection area between the wires and the ITO-plated $\mathrm{LiNbO}_{3}$ blocked light penetration (see Fig. 1d, i.e., the dark regions in the aperture). Hence, the transducer's light transmittance can be considerably improved when either small size or focused light is used for imaging. Beam spreading due to the acoustic lens is not a problem for ARPAM because its imaging resolution is mainly determined by an US transducer. Note that the light energy loss in ITOplated $\mathrm{LiNbO}_{3}$ can be mitigated by increasing the center frequency of the transducer since the thickness of the active layer decreases as the center frequency increases [40].

As shown in Fig. 5, the lateral resolution of PA imaging is typically inferior to that of US imaging. This is because the US image can be constructed using US waves focused on both transmission and reception. This two-way focusing is possible when a target is placed at the focal point of a transducer. In PA imaging, however, only focusing for reception is available, referred to as one-way focusing. As a result, the sidelobes of PA images are typically doubled compared to those of US images. To improve the lateral resolution of PA images, adaptive receive beamforming and synthetic focusing can be used [27, 41-43], which was previously proposed to increase the lateral resolution of US images.

The developed transducer was initially designed for ARPAM and combined PA-US imaging. To achieve this goal, the optically transparent acoustic lens was developed. In addition, the backing layer and long housing were designed for easy integration of the transducer with an optical fiber bundle. However, this proposed structure for transparent transducers is also viable for OR-PAM. In this case, the backing layer should be designed to have a plano-convex shape for light focusing, and the central area of the acoustic lens equal to the light beam width should be flattened to serve as a plane-parallel plate if collimated light is irradiated. When focused light is used, the backing layer and the central area of the acoustic lens should adopt the shape of a plane-parallel plate. Note that the flat central area of the acoustic lens can be determined to avoid affecting US focusing performance because US wavelengths are longer than those of light, and US waves generated within a given area have a similar phase. A transparent transducer with such a 

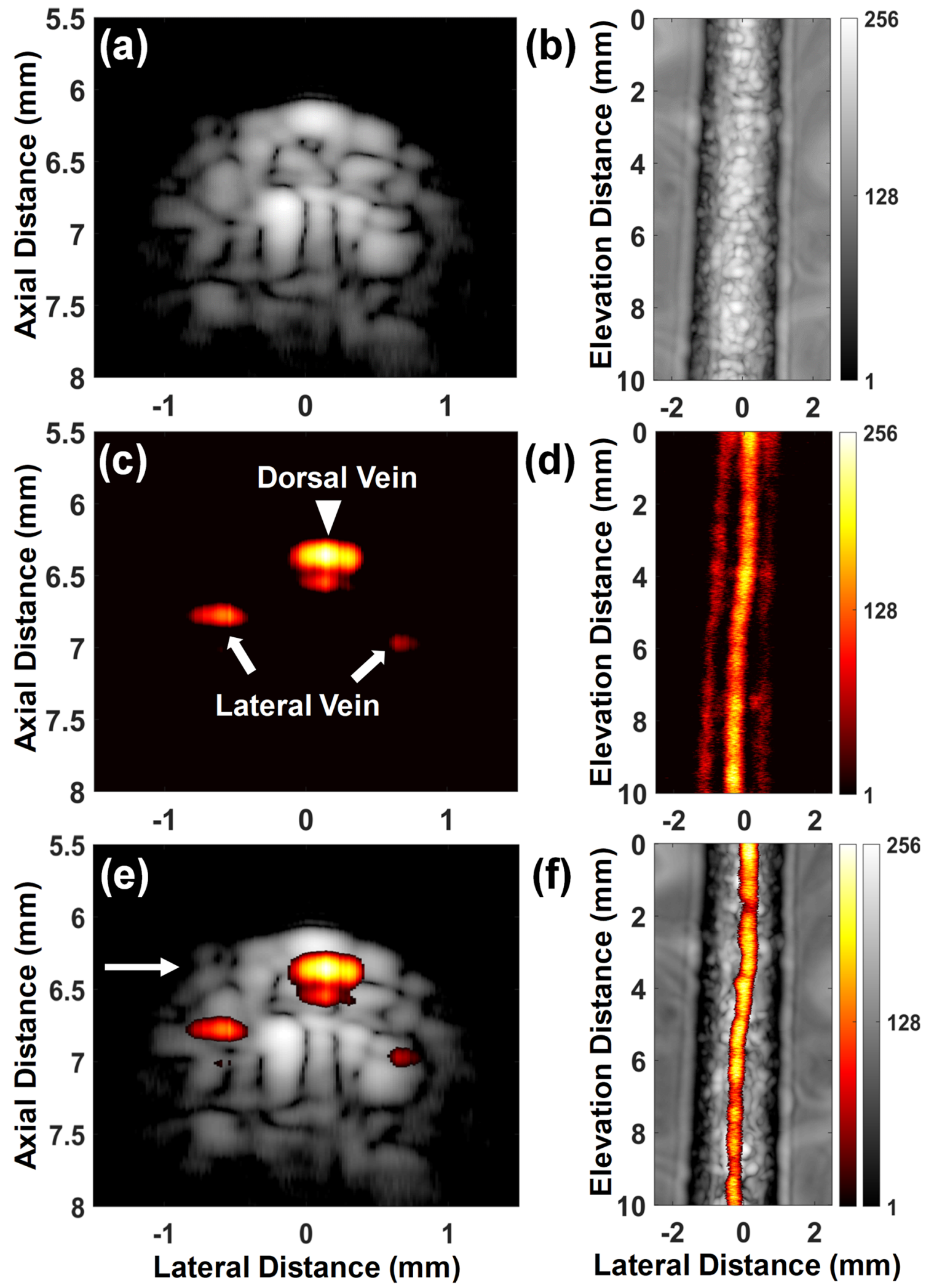

Fig. 7 In vivo imaging. a, $\mathbf{c}$ Cross-section and $\mathbf{b}, \mathbf{d}$ maximum intensity projection US and PA images of a mouse tail. Combined US and PA images in $\mathbf{e}$ the axial-lateral imaging plane and $\mathbf{f}$ the elevation-

lateral imaging plane at an axial depth of $6.43 \mathrm{~mm}$ indicated by the white arrow in (e) 
structure is currently under development, intended for use with OR-PAM, AR-PAM, and combined PA-US microscopy.

\section{Conclusion}

In this paper, we have proposed an optically transparent focused US transducer consisting of optically transparent acoustic stacks, i.e., ITO-plated $\mathrm{LiNbO}_{3}$, a backing layer, and an acoustic lens. The transducer's optical properties enabled simple integration of optical and acoustic modules for AR-PAM and combined PA-US imaging. Results from in vitro and in vivo experiments demonstrated the transducer to be suitable for both US and PA imaging. The relatively narrow bandwidth of the transducer, occurring due to no acoustic matching layers, limits the axial resolution. Use of $1-3$ or $2-2$ piezocomposites could offer a viable solution to the problem unless optically transparent materials for acoustic matching layers are found $[6,44]$. The transducer's center frequency should be increased to improve spatial resolution, particularly for AR-PAM used in biological applications. The increase in center frequency leads to a reduction in thickness and size of the active layer, which is advantageous for increasing light transmittance but requires sophisticated acoustic lens and signal wiring design. In conclusion, the results of this study represent a step toward efficient construction of probes for combined PA and US microscopy.

Acknowledgements This work was supported by the Samsung Research Funding Center of Samsung Electronics under Project Number SRFC-IT1702-03.

Open Access This article is licensed under a Creative Commons Attribution 4.0 International License, which permits use, sharing, adaptation, distribution and reproduction in any medium or format, as long as you give appropriate credit to the original author(s) and the source, provide a link to the Creative Commons licence, and indicate if changes were made. The images or other third party material in this article are included in the article's Creative Commons licence, unless indicated otherwise in a credit line to the material. If material is not included in the article's Creative Commons licence and your intended use is not permitted by statutory regulation or exceeds the permitted use, you will need to obtain permission directly from the copyright holder. To view a copy of this licence, visit http://creativecommons.org/licenses/by/4.0/.

\section{References}

1. Wang, L. V., \& Hu, S. (2012). Photoacoustic tomography: In vivo imaging from organelles to organs. Science, 335(6075), $1458-1462$.

2. Strohm, E. M., Berndl, E. S. L., \& Kolios, M. C. (2013). High frequency label-free photoacoustic microscopy of single cells. Photoacoustics, 1(3-4), 49-53.
3. Wong, T. T. W., Zhang, R., Zhang, C., Hsu, H.-C., Maslov, K. I., Wang, L., ... Wang, L. V. (2017). Label-free automated threedimensional imaging of whole organs by microtomy-assisted photoacoustic microscopy. Nature Communications, 8(1), 1386.

4. Wong, T. T. W., Zhang, R., Hai, P., Zhang, C., Pleitez, M. A., Aft, R. L., ... Wang, L. V. (2017). Fast label-free multilayered histology-like imaging of human breast cancer by photoacoustic microscopy. Science Advances, 3(5), e1602168.

5. Liu, W., \& Yao, J. (2018). Photoacoustic microscopy: Principles and biomedical applications. Biomedical Engineering Letters, 8(2), 203-213.

6. Jang, J., \& Chang, J. H. (2018). Design and fabrication of a miniaturized convex array for combined ultrasound and photoacoustic imaging of the prostate. IEEE Transactions on Ultrasonics, Ferroelectrics, and Frequency Control, 65(11), 2086-2096.

7. Choi, W., Park, E.-Y., Jeon, S., \& Kim, C. (2018). Clinical photoacoustic imaging platforms. Biomedical Engineering Letters, $8(2), 139-155$.

8. Zhang, H. F., Maslov, K., Stoica, G., \& Wang, L. V. (2006). Functional photoacoustic microscopy for high-resolution and noninvasive in vivo imaging. Nature Biotechnology, 24(7), $848-851$.

9. Lan, B., Liu, W., Wang, Y., Shi, J., Li, Y., Xu, S., ... Yao, J. (2018). High-speed widefield photoacoustic microscopy of smallanimal hemodynamics. Biomedical Optics Express, 9(10), 4689.

10. Kim, H. H., Cannata, J. M., Liu, R., Chang, J. H., Silverman, R. H., \& Shung, K. K. (2008). $20 \mathrm{MHz} / 40 \mathrm{MHz}$ dual element transducers for high frequency harmonic imaging. IEEE Transactions on Ultrasonics, Ferroelectrics, and Frequency Control, 55(12), 2683-2691.

11. Park, J., Lee, C., Lee, J., Ha, J.-I., Choi, H., \& Chang, J. H. (2019). Magnetically actuated forward-looking interventional ultrasound imaging: Feasibility studies. IEEE Transactions on Biomedical Engineering. https://doi.org/10.1109/TBME.2019.2948391

12. Jeong, M. K., \& Kwon, S. J. (2018). Side lobe free medical ultrasonic imaging with application to assessing side lobe suppression filter. Biomedical Engineering Letters, 8(4), 355-364.

13. Yao, J., \& Wang, L. V. (2014). Sensitivity of photoacoustic microscopy. Photoacoustics, 2(2), 87-101.

14. Hu, S., Maslov, K., \& Wang, L. V. (2011). Second-generation optical-resolution photoacoustic microscopy with improved sensitivity and speed. Optics Letters, 36(7), 1134.

15. Zhang, C., Maslov, K., Hu, S., Chen, R., Zhou, Q., Shung, K. K., \& Wang, L. V. (2012). Reflection-mode submicron-resolution in vivo photoacoustic microscopy. Journal of Biomedical Optics, 17(2), 020501.

16. Wang, L., Maslov, K., \& Wang, L. V. (2013). Single-cell label-free photoacoustic flowoxigraphy in vivo. Proceedings of the National Academy of Sciences of the United States of America, 110(15), 5759-5764.

17. Cannata, J. M., Ritter, T. A., Chen, Wo.-Hs.ing, Silverman, R. H., \& Shung, K. K. (2003). Design of efficient, broadband singleelement $(20-80 \mathrm{MHz})$ ultrasonic transducers for medical imaging applications. IEEE Transactions on Ultrasonics, Ferroelectrics and Frequency Control, 50(11), 1548-1557.

18. Brodie, G. W. J., Qiu, Y., Cochran, S., Spalding, G. C., \& Macdonald, M. P. (2014). Optically transparent piezoelectric transducer for ultrasonic particle manipulation. IEEE Transactions on Ultrasonics, Ferroelectrics, and Frequency Control, 61(3), 389-391.

19. Liu, H., Avrutin, V., Izyumskaya, N., Özgür, Ü, \& Morkoç, H. (2010). Transparent conducting oxides for electrode applications in light emitting and absorbing devices. Superlattices and Microstructures, 48(5), 458-484.

20. Pang, D. C., \& Chang, C. M. (2017). Development of a novel transparent flexible capacitive micromachined ultrasonic transducer. Sensors (Basel, Switzerland), 17(6), 1443. 
21. Li, Z., Ilkhechi, A. K., \& Zemp, R. (2019). Transparent capacitive micromachined ultrasonic transducers (CMUTs) for photoacoustic applications. Optics Express, 27(9), 13204.

22. Dangi, A., Agrawal, S., \& Kothapalli, S.-R. (2019). Lithium niobate-based transparent ultrasound transducers for photoacoustic imaging. Optics Letters, 44(21), 5326.

23. Chen, H., Agrawal, S., Dangi, A., Wible, C., Osman, M., Abune, L., \& Kothapalli, S. (2019). Optical-resolution photoacoustic microscopy using transparent ultrasound transducer. Sensors (Basel, Switzerland), 19(24), 5470.

24. Lee, J., \& Chang, J. H. (2019). Dual-element intravascular ultrasound transducer for tissue harmonic imaging and frequency compounding: Development and imaging performance assessment. IEEE Transactions on Biomedical Engineering, 66(11), 3146-3155.

25. Fang, C., Hu, H., \& Zou, J. (2020). A focused optically transparent PVDF transducer for photoacoustic microscopy. IEEE Sensors Journal, 20(5), 2313-2319.

26. Snook, K. A., Zhao, J.-Z., Alves, C. H. F., Cannata, J. M., Chen, W.-H., Meyer, R. J., ... Shung, K. K. (2002). Design, fabrication, and evaluation of high frequency, single-element transducers incorporating different materials. IEEE Transactions on Ultrasonics, Ferroelectrics and Frequency Control, 49(2), 169-176.

27. Yoon, C., Yoo, Y., Song, T., \& Chang, J. H. (2014). Pixel based focusing for photoacoustic and ultrasound dual-modality imaging. Ultrasonics, 54(8), 2126-2133.

28. Kim, H., \& Chang, J. H. (2018). Multimodal photoacoustic imaging as a tool for sentinel lymph node identification and biopsy guidance. Biomedical Engineering Letters, 8(2), 183-191.

29. Kim, H., Jo, G., \& Chang, J. H. (2018). Ultrasound-assisted photothermal therapy and real-time treatment monitoring. Biomedical Optics Express, 9(9), 4472-4480.

30. Tang, Y., Liu, W., Li, Y., Zhou, Q., \& Yao, J. (2018). Concurrent photoacoustic and ultrasound microscopy with a coaxial dualelement ultrasonic transducer. Visual Computing for Industry, Biomedicine, and Art, 1(1), 3.

31. Strohm, E. M., Moore, M. J., \& Kolios, M. C. (2016). High resolution ultrasound and photoacoustic imaging of single cells. Photoacoustics, 4(1), 36-42.

32. Jang, J., \& Chang, J. H. (2016). Design and fabrication of doublefocused ultrasound transducers to achieve tight focusing. Sensors (Basel, Switzerland), 16(8), 1248.

33. Foster, F. S., Pavlin, C. J., Harasiewicz, K. A., Christopher, D. A., \& Turnbull, D. H. (2000). Advances in ultrasound biomicroscopy. Ultrasound in Medicine \& Biology, 26(1), 1-27.

34. Kang, J., Chang, J. H., Kim, S. M., Lee, H. J., Kim, H., Wilson, B. C., \& Song, T.-K. (2017). Real-time sentinel lymph node biopsy guidance using combined ultrasound, photoacoustic, fluorescence imaging: In vivo proof-of-principle and validation with nodal obstruction. Scientific Reports, 7(1), 45008.

35. Staszyk, C., Bohnet, W., Gasse, H., \& Hackbarth, H. (2003). Blood vessels of the rat tail: A histological re-examination with respect to blood vessel puncture methods. Laboratory Animals, $37(2), 121-125$

36. Moon, J.-Y., Lee, J., \& Chang, J. H. (2016). Electrical impedance matching networks based on filter structures for high frequency ultrasound transducers. Sensors and Actuators A: Physical, 251, 225-233.

37. Rathod, V. T. (2019). A review of electric impedance matching techniques for piezoelectric sensors, actuators and transducers. Electronics, 8(2), 169.

38. McKeighen, R. E. (1998). Design guidelines for medical ultrasonic arrays. In K. K. Shung (Ed.), Medical imaging 1998: Ultrasonic transducer engineering (pp. 2-18). Bellingham: SPIE.

39. Sherar, M. D., \& Foster, F. S. (1989). The design and fabrication of high frequency poly(vinylidene fluoride) transducers. Ultrasonic Imaging, 11(2), 75-94.

40. Lee, J., Moon, J.-Y., \& Chang, J. (2018). A 35 MHz/105 MHz dual-element focused transducer for intravascular ultrasound tissue imaging using the third harmonic. Sensors (Basel, Switzerland), 18(7), 2290.

41. Jeon, S., Park, J., Managuli, R., \& Kim, C. (2019). A novel 2-D synthetic aperture focusing technique for acoustic-resolution photoacoustic microscopy. IEEE Transactions on Medical Imaging, $38(1), 250-260$

42. Chang, J. H., \& Song, T.-K. (2011). A new synthetic aperture focusing method to suppress the diffraction of ultrasound. IEEE Transactions on Ultrasonics, Ferroelectrics and Frequency Control, 58(2), 327-337.

43. Yoon, C., Kang, J., Han, S., Yoo, Y., Song, T.-K., \& Chang, J. H. (2012). Enhancement of photoacoustic image quality by sound speed correction: Ex vivo evaluation. Optics Express, 20(3), 3082.

44. Cha, J. H., \& Chang, J. H. (2014). Development of 15 MHz 2-2 piezo-composite ultrasound linear array transducers for ophthalmic imaging. Sensors and Actuators A: Physical, 217, 39-48.

Publisher's Note Springer Nature remains neutral with regard to jurisdictional claims in published maps and institutional affiliations. 